\title{
Absence of Distinctively High Grain-Boundary Impedance in Polycrystalline Cubic Bismuth Oxide
}

\author{
Hyun Joon Jung and Sung-Yoon Chung ${ }^{\dagger}$ \\ Graduate School of EEWS, KAIST, Daejeon 34141, Korea
}

(Received June 26, 2017; Revised August 7, 2017; Accepted August 8, 2017)

\begin{abstract}
In this work, we studied a fluorite structure oxides: Yttria stabilized zirconia, (YSZ); Gd doped $\mathrm{CeO}_{2}$ (GDC); erbia stabilized $\mathrm{Bi}_{2} \mathrm{O}_{3}$ (ESB); $\mathrm{Zr}$ doped erbia stabilized $\mathrm{Bi}_{2} \mathrm{O}_{3}$ (ZESB); Ca doped erbia stabilized $\mathrm{Bi}_{2} \mathrm{O}_{3}$ (CESB) in the temperature range of 250 to $600^{\circ} \mathrm{C}$ using electrochemical impedance spectroscopy (EIS). As is well known, grain boundary blocking effect was observed in YSZ and GDC. However, there is no grain boundary effect on ESB, ZESB, and CESB. The Nyquist plots of these materials exhibit a single arc at low temperature. This means that there is no space charge effect on $\delta-\mathrm{Bi}_{2} \mathrm{O}_{3}$. In addition, impedance data were analyzed by using the brick layer model. We indirectly demonstrate that grain boundary ionic conductivity is similar to or even higher than bulk ionic conductivity on cubic bismuth oxide.
\end{abstract}

Key words : Cubic bismuth oxide, Grain boundary blocking effect, Space charge, Electrochemical impedance spectroscopy

\section{Introduction}

$\delta$ $-\mathrm{Bi}_{2} \mathrm{O}_{3}$ is drawing much attention as the most wellknown material among oxygen ionic conductors. ${ }^{1-5)}$ The attention is due to the high ionic conductivity of $\delta-\mathrm{Bi}_{2} \mathrm{O}_{3}$. The ionic conductivity of $\delta-\mathrm{Bi}_{2} \mathrm{O}_{3}$ is higher than that of yttria stabilized zirconia (YSZ) $\left(5 \times 10^{-3} \mathrm{~S} / \mathrm{cm}\right.$ at $\left.600^{\circ} \mathrm{C}\right)$, which is frequently used as an oxygen ionic conductor, by two orders of magnitude and is higher than that of gadolinium doped ceria (GDC) $\left(2.5 \times 10^{-2} \mathrm{~S} / \mathrm{cm}\right.$ at $\left.600^{\circ} \mathrm{C}\right)$ by one order of magnitude. ${ }^{6-7)}$ The high ionic conductivity of $\delta-\mathrm{Bi}_{2} \mathrm{O}_{3}$ is due to the oxygen vacancy concentration (25\%) and the anion mobility in $\delta-\mathrm{Bi}_{2} \mathrm{O}_{3}$ which values are higher than those of acceptordoped ceria or acceptor-doped zirconia having the same fluorite-type structure (Fig. 1) ${ }^{8)}$ However, the overall ionic conductivity of actual polycrystals is determined by not only the bulk ionic conductivity but also the ionic conductivity of the grain boundaries. According to Guo, ${ }^{9)}$ in acceptor-doped zirconia and acceptor-doped ceria, which are materials having a fluorite-type structure like that of $\delta-\mathrm{Bi}_{2} \mathrm{O}_{3}$, the ionic conductivity at the grain boundaries is several orders of magnitude lower than the bulk ionic conductivity, which is called the grain boundary blocking effect. ${ }^{10-14)}$ These two factors are often mentioned as the causes of the grain boundary blocking effect. First, grain boundary siliceous phases impede ionic conduction. ${ }^{13-14)}$ However, the grain boundary blocking effect is even found in high-purity materials. ${ }^{12-15)}$ This blocking nature can be accounted by the presence of a

Corresponding author : Sung-Yoon Chung

E-mail : sychung@kaist.ac.kr

Tel : +82-42-350-1723 Fax : +82-42-350-1710 space charge layer near the grain boundary core. ${ }^{12,16-17)}$ Many experiment results support a role of space charge. Lei used transmission electron microscopy (TEM) and electron energy loss spectroscopy (EELS) to determine an increase of oxygen vacancy and $\mathrm{Y}$ segregation at the grain boundary core of YSZ and also demonstrated that Gd segregation and oxygen vacancy were higher at the grain boundaries than in the bulk GDC. ${ }^{18)}$ Lee directly observed the Gd segregation and high concentration of oxygen vacancy near grain boundaries using scanning transmission electron microscopy-Xray spectroscopy (STEM-EDS). In addition, in this work, Kelvin probe force microscopy (KFM) verified Grain boundary is positively charged and the potential difference between grain boundary and bulk. Moreover, the potential difference was equal to the calculated data from impedance analysis applying the Schottky barrier model. ${ }^{19)}$ However, same fluorite-type structure $\delta-\mathrm{Bi}_{2} \mathrm{O}_{3}$ didn't show grain boundary effect. ${ }^{20)}$ In this work we added an aliovalent dopant to $\delta-\mathrm{Bi}_{2} \mathrm{O}_{3}$, as it was done in YSZ and GDC to know whether grain boundary effect shows or not. The difference of the ionic radius makes an elastic filed and it causes the segregation in the grain boundary. ${ }^{21)}$ So we selected a dopant which has a small ionic radius difference with $\mathrm{Bi}_{2} \mathrm{O}_{3}$ $\left(\mathrm{r}\left[\mathrm{Bi}^{3}\right]=1.17 \AA\right)^{22)}$ to exclude the segregation effect as much as possible. A tetravalent donor $\left.\mathrm{ZrO}_{2}\left(\mathrm{r}_{2}\left[\mathrm{Zr}^{4+}=0.84 \AA\right] \mathrm{I}\right]\right)^{22)}$ and a divalent acceptor $\mathrm{CaO}\left(\mathrm{r}\left[\mathrm{Ca}^{2+}=1.12 \AA\right]\right)^{22)}$ were doped to $\mathrm{Bi}_{2} \mathrm{O}_{3}$ to investigate the grain boundary blocking effect caused by the space charge layer. Using Kröger-Vink notation, ${ }^{23)}$ we describe a doping $\mathrm{Bi}_{2} \mathrm{O}_{3}$ with donor and acceptor, as follows:

$$
2 \mathrm{ZrO}_{2}+\mathrm{V}_{\mathrm{O}}^{*} \rightarrow 2 \mathrm{Zr}_{\mathrm{Bi}}^{\cdot}+4 \mathrm{O}_{\mathrm{O}}^{\mathrm{X}}
$$




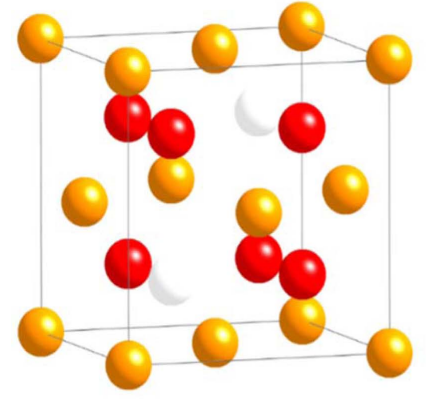

(a)

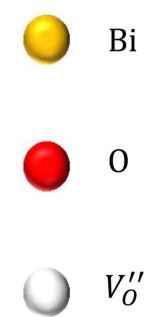

$\mathrm{Gd}_{2} \mathrm{O}_{3} \stackrel{\mathrm{CeO}_{2}}{\longrightarrow} 2 \mathrm{Gd}_{c e}^{\prime}+3 \mathrm{O}_{O}^{X}+V_{o}^{*}$

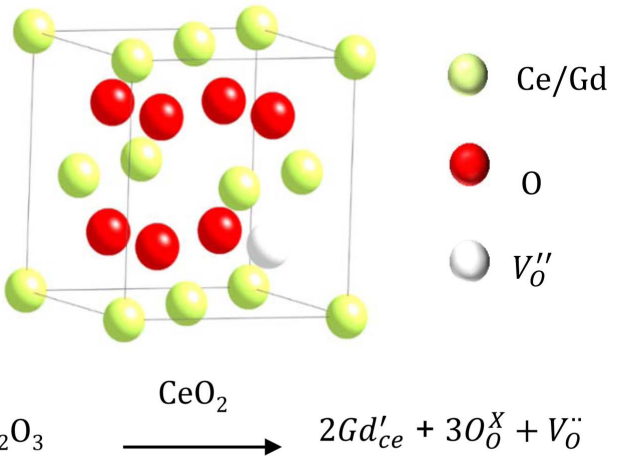

(b)

Fig. 1. Crystal structure of (a) $\delta-\mathrm{Bi}_{2} \mathrm{O}_{3}$, (b) Gd doped ceria. Yttria stabilized zirconia (YSZ) has the same structure as (b).

$$
2 \mathrm{CaO} \rightarrow 2 \mathrm{Ca}_{\mathrm{Bi}}^{\prime}+\mathrm{V}_{\mathrm{O}}^{\ddot{*}}+2 \mathrm{O}_{\mathrm{O}}^{\mathrm{X}}
$$

Doping with the donor $\mathrm{Zr}$ was expected to lead to a positively charged space charge layer because of $\mathrm{Zr}_{\mathrm{Bi}}$ segregation at the grain boundary (Eq. 1a), while the doping with the acceptor $\mathrm{Ca}$ was expected to generate a negatively charged space charge layer because of the $\mathrm{Ca}_{\mathrm{Bi}}^{\prime}$ segregation at the grain boundary (Eq. $1 b$ ). Since $\delta-\mathrm{Bi}_{2} \mathrm{O}_{3}$ is converted to $\alpha-\mathrm{Bi}_{2} \mathrm{O}_{3}$ at $723^{\circ} \mathrm{C},{ }^{24)}$ for stabilization at room temperature, we doped $\delta-\mathrm{Bi}_{2} \mathrm{O}_{3}$ with $20 \mathrm{~mol} \%$ Er. We call this erbia-stabilized bismuth (ESB), $\mathrm{Bi}_{0.8} \mathrm{Er}_{0.2} \mathrm{O}_{1.5}$. In addition, $2 \mathrm{~mol} \% \mathrm{Ca}$ and $5 \mathrm{~mol} \% \mathrm{Zr}$ were doped into the ESB. Polycrystal $8 \mathrm{~mol} \%$ $\mathrm{Y}$ doped $\mathrm{ZrO}_{2}$, single-crystal $8 \mathrm{~mol} \% \mathrm{Y}$ doped $\mathrm{ZrO}_{2}$, and 10 mol\% Gd doped $\mathrm{CeO}_{2}$, which also have fluorite structure were used for comparison. In addition, we perform X-ray diffraction (XRD) analysis to identify synthesized materials. Microstructures of the samples were analyzed by scanning electron microscopy (SEM) to verify the absence of a secondary phase at the grain boundary. The ionic conductivity of the bulk and the grain boundaries is measured with the electrochemical impedance spectroscopy.

\section{Experimental Procedure}

$\mathrm{Bi}_{0.8} \mathrm{Er}_{0.2} \mathrm{O}_{1.5}$ (ESB) was synthesized by conventional solidstate synthesis using $\mathrm{Bi}_{2} \mathrm{O}_{3}\left(99.8 \%\right.$, Aldrich) and $\mathrm{Er}_{2} \mathrm{O}_{3}(99.99 \%$, Aldrich) powders. The powders were Ball-milled for $24 \mathrm{~h}$ using zirconia balls, and then the powders were sieved using a 300 mesh sieve. Subsequently, the resulting powders were calcinated at $800^{\circ} \mathrm{C}$ for $10 \mathrm{~h}$. The $\left(\mathrm{Bi}_{0.8} \mathrm{Er}_{0.2} \mathrm{O}_{1.5}\right)_{0.95^{-}}$ $\left(\mathrm{ZrO}_{2}\right)_{0.05}(\mathrm{ZESB})$ powder doped with $5 \mathrm{~mol} \% \mathrm{Zr}$ was synthesized by the same method using $\mathrm{Zr}_{2} \mathrm{O}_{3}$ (99.99\%, Aldrich) powder as a dopant. $\left(\mathrm{Bi}_{0.8} \mathrm{Er}_{0.2} \mathrm{O}_{1.5}\right)_{0.98}(\mathrm{CaO})_{0.02}$ (CESB) powder doped with $2 \mathrm{~mol} \% \mathrm{Ca}$ was synthesized by the same method using $\mathrm{CaCO}_{3}(99.99 \%$, Aldrich) as a dopant. X-ray diffraction (XRD, RIGAKU D/MAX-2500) was used to verify if the powders were well synthesized, and X-ray diffraction pattern analysis in the $2 \theta$ range from $20^{\circ}$ to $80^{\circ}$ was obtained using $\mathrm{Cu} K_{\alpha 1}\left(\lambda_{\mathrm{CuK \alpha}}=1.5406 \AA\right.$ at room temperature. Pellets having a diameter of $10 \mathrm{~mm}$ were prepared by using a calcinated powder. The pellet were uniaxial pressed using Cold isostatic pressing (CIP) at $200 \mathrm{MPa}$. Then the pellet was sintered in air at $890^{\circ} \mathrm{C}$ for $16 \mathrm{~h}$. $\mathrm{Ce}_{0.9} \mathrm{Gd}_{0.1} \mathrm{O}_{1,95}$ (GDC) powder was purchased from Aldrich. Pellets having a diameter of $10 \mathrm{~mm}$ were prepared by using a GDC powder. The pellet were uniaxial pressed using CIP at $200 \mathrm{MPa}$. and the sample was sintered at $1500^{\circ} \mathrm{C}$ for $12 \mathrm{~h} .8 \mathrm{~mol} \% \mathrm{Y}$ doped $\mathrm{ZrO}_{2}$ (YSZ) polycrystalline substrate with a size of $1 \mathrm{~cm} \times 1 \mathrm{~cm} \times$ $0.5 \mathrm{~cm}$ and a $8 \mathrm{~mol} \% \mathrm{Y}$ doped $\mathrm{ZrO}_{2}$ (YSZ) single-crystal [100] substrate with a size of $1 \mathrm{~cm} \times 1 \mathrm{~cm} \times 0.5 \mathrm{~cm}$ were used; both were purchased from MTI KOREA. To measure the impedance spectra, Pt paste was applied on both sides of the pellets, and then samples were sintered at $800^{\circ} \mathrm{C}$ for $2 \mathrm{~h}$ in air to make electrodes. The ionic conductivity of the samples was measured by using an EIS (VSP-300, Biologic) over a frequency range of $1 \mathrm{~Hz}$ to $5 \mathrm{MHz}$ with a voltage amplitude of $50 \mathrm{mV}$. The measurement data were analyzed using the EC-Lab software program (ver. 11.02). For the analysis of the microstructure, all samples were thermally etched at $800^{\circ} \mathrm{C}$ for one minute and GDC were thermally etched at $1300^{\circ} \mathrm{C}$ for one minute, and the microstructures were observed using SEM (Hitachi SU5000).

\section{Results and Discussion}

\subsection{Microstructural and structural characteriza- tion}

A cubic crystal structure (space group of $\mathrm{Fm}-3 \mathrm{~m}$ ) $\delta-\mathrm{Bi}_{2} \mathrm{O}_{3}$ has a stable phase from $723^{\circ} \mathrm{C}$ to $825^{\circ} \mathrm{C}$, the melting point. ${ }^{24)}$ On cooling below $723^{\circ} \mathrm{C}, \delta-\mathrm{Bi}_{2} \mathrm{O}_{3}$ is transformed to monoclinic $\mathrm{Bi}_{2} \mathrm{O}_{3}$. Doping the cubic $\delta-\mathrm{Bi}_{2} \mathrm{O}_{3}$ with cations with high polarizability is known as an effective method of stabilizing the cubic $\delta-\mathrm{Bi}_{2} \mathrm{O}_{3}$ phase. Among the high polarizability cations, Er has a relatively small ionic radius and can stabilize $\delta-\mathrm{Bi}_{2} \mathrm{O}_{3}$ with a minimum amount of dopant. Doping $\delta-\mathrm{Bi}_{2} \mathrm{O}_{3}$ with $20 \mathrm{~mol} \% \mathrm{Er}$ is the optimal doping concentration and showed the highest ionic conductivity. ${ }^{25)}$ In this work, ESB was doped with $2 \mathrm{~mol} \% \mathrm{Ca}$. this is not at the same concen- 


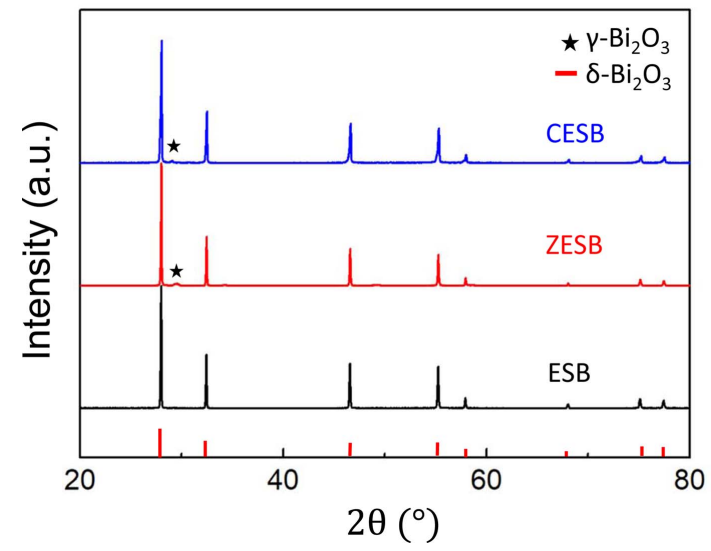

Fig. 2. Powder X-ray diffraction patterns of ESB, ZESB and CESB after $800^{\circ} \mathrm{C}$ calcination with index from ICDD card 00-034-0377, 03-065-3319.

tration of the zirconia dopant, $5 \mathrm{~mol} \%$. Because the solubility of $\mathrm{Ca}$ in $\mathrm{ESB}$ is lower than $5 \mathrm{~mol} \%$. XRD was performed to characterize the ESB, CESB, and ZESB powders calci- nated at $800^{\circ} \mathrm{C}$ for $10 \mathrm{~h}$. The XRD data showed that all the peaks of the ESB, CESB, and ZESB powders were well matched with the peak of $\delta-\mathrm{Bi}_{2} \mathrm{O}_{3}$, which had a cubic crystal structure (ICDD card 00-034-0377; a=5.479 ^) (Fig. 2). A small amount of $\gamma-\mathrm{Bi}_{2} \mathrm{O}_{3}$, formed from slow cooling of $\delta$ $\mathrm{Bi}_{2} \mathrm{O}_{3}$, was detected in the ZESB and CESB powders; no other secondary phase was found. Size of the grains can be obtained from the cross-sectional SEM images; the images showed no secondary phase at the grain boundaries (Fig. 3). In addition, the SEM images also confirmed that the singlecrystal YSZ has only one grain without a grain boundary, and confirmed that all the samples were sintered densely.

\subsection{Brick layer model}

Electrochemical Impedance spectroscopy is a very useful measurement method because the conductivity of the bulk and the grain boundaries can be separately measured in a polycrystalline material. In this study, the brick layer model was applied for impedance spectra analysis ${ }^{27-29)}$ The description of the brick layer model is given a review article by Haile. ${ }^{27)}$ Fig. 4 shows the general circuit and a typical
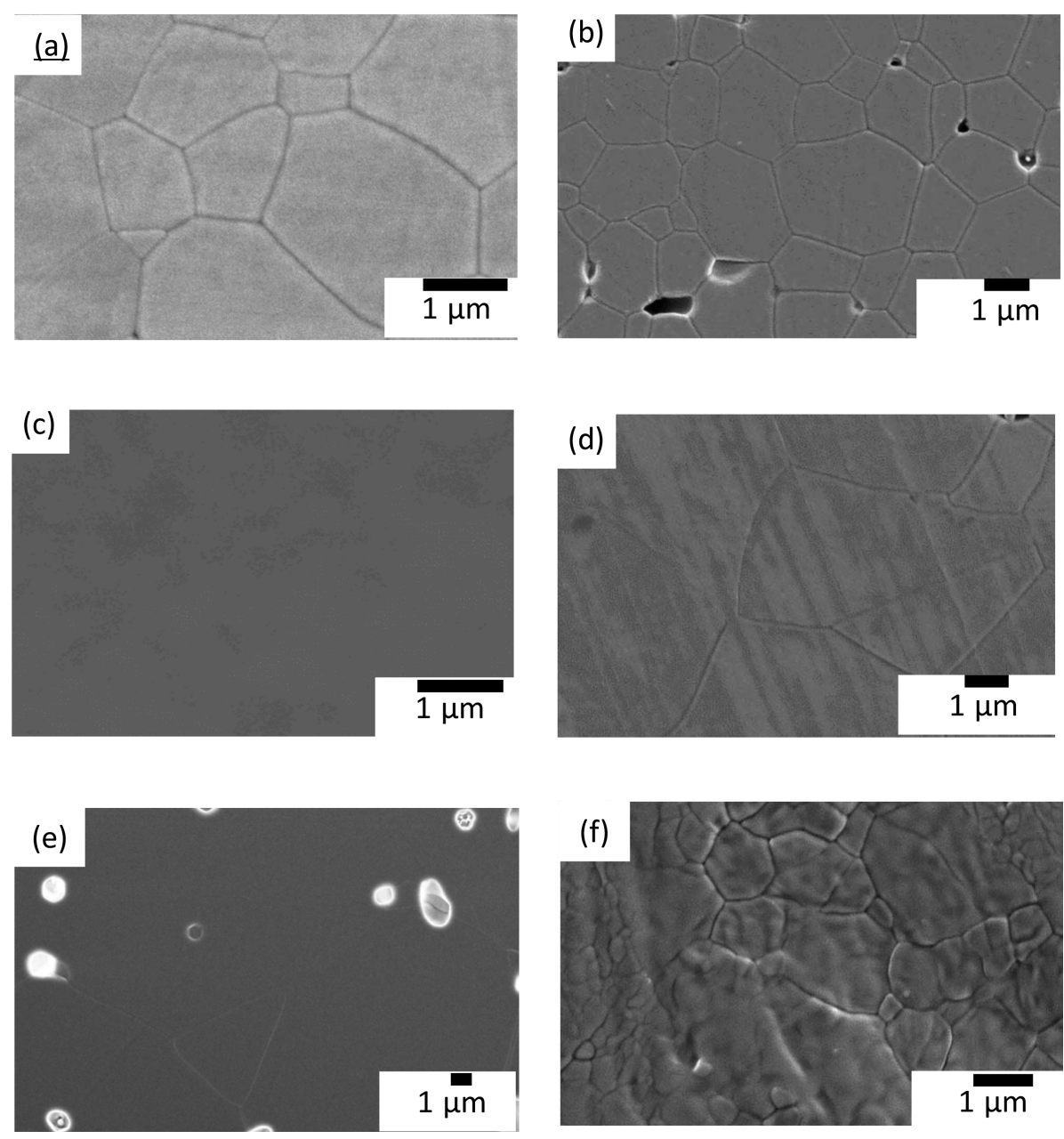

Fig. 3. SEM image showing the cross section microstructures: (a) GDC with average grain size of $1.65 \mu \mathrm{m}$ (b) polycrystalline YSZ with average grain size of $2.85 \mu \mathrm{m}$ (c) single crystal YSZ, (d) ESB with average grain size of $6.51 \mu \mathrm{m}$ (e) ZESB with average grain size of $4.1 \mu \mathrm{m}$ (f) CESB with average grain size of $2.3 \mu \mathrm{m}$. 


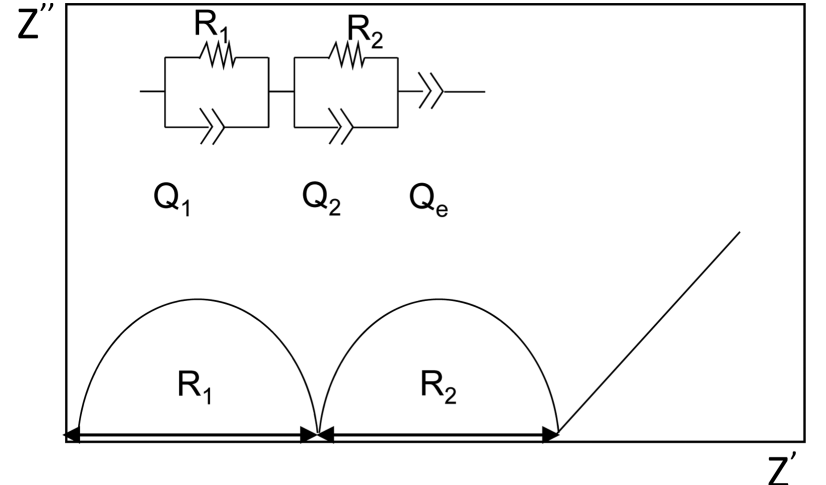

Fig. 4. A typical Nyquist plot and equivalent circuit of a polycrystalline material with brick layer model. $\mathrm{Q}$ is the constant phase element and $\mathrm{R}$ is the resistance.

Nyquist plot of polycrystals. The Nyquist plot is a plot in which the $\mathrm{x}$-axis represents the real part of the impedance and the $y$-axis is the imaginary part of the impedance. The circuit consists of three RQ sub-circuits, wherein $\mathrm{R}\left(Z_{R}=\mathrm{R}\right)$ denotes the resistance and $\mathrm{Q}$ the constant phase element (CPE). $Z_{Q}=\left[Y\left(j w^{n}\right)\right]^{-1}$, wherein $\mathrm{j}=\sqrt{-1}, \omega$ is the frequency, $\mathrm{Y}$ and $\mathrm{n}$ are constants, and $\mathrm{n}$ is a value between 0 and 1 . If $\mathrm{n}$, which experimentally determines the characteristics of the RQ sub-circuits, is close to 1 , the constant phase elements is similar to a capacitor, and Y is called the pseudocapacitance. The frequency at the vertex of the semicircle shown in Fig. 4 is referred to as the characteristic frequency, $w_{0}$, of the sub-circuit $\mathrm{RQ}$, which is expressed as $(Y R)^{-1 / n}$. If the frequency of that circuit is sufficiently different from another and $\mathrm{n}$ is close to 1 , the resistance of each circuit can be calculated from the size of the arc on the real axis, and the capacitance of the sub-circuit is given as $\left(R w_{0}\right)^{-1}$. The resistance values are $R_{1}$ and $R_{2}$ of the RQ circuit. To associate these values with physical meanings, a cubic grain

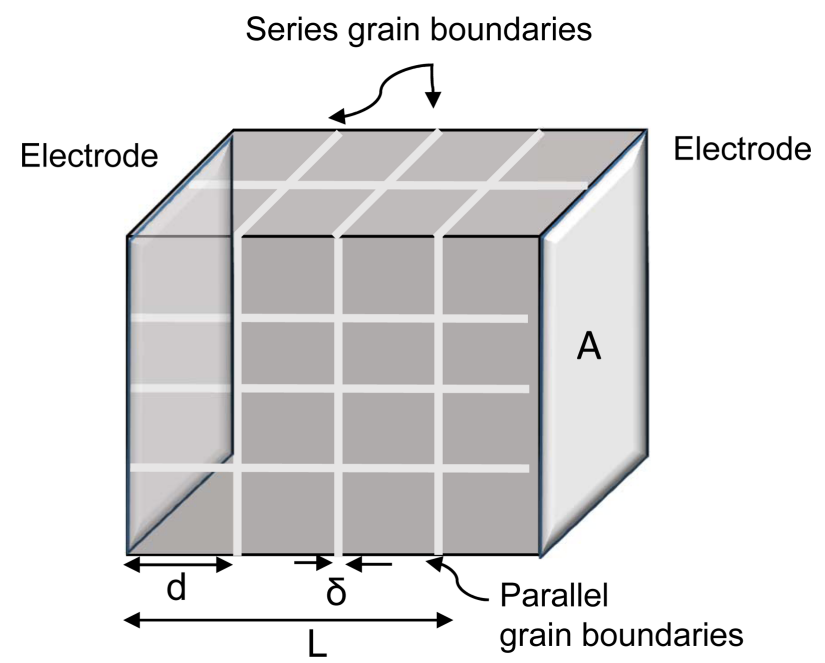

Fig. 5. "Brick layer" model of a polycrystalline material. Grains are assumed to be cube-shaped, and grain boundaries exist as flat layers between grains. ${ }^{29}$

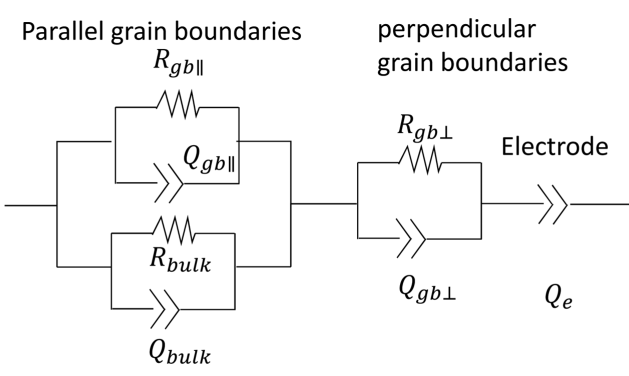

Grain interiors(bulk)

(a)

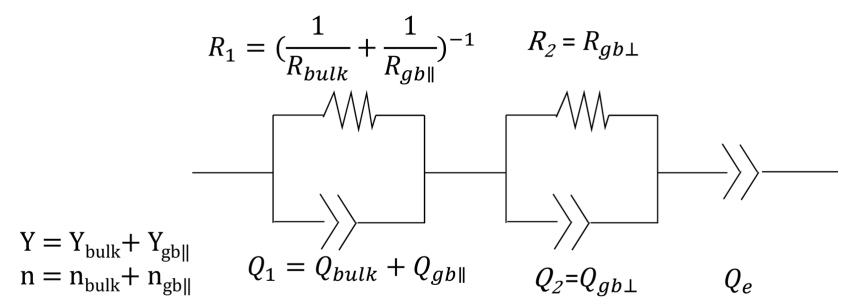

(b)

Fig. 6. Equivalent circuit models of a polycrystalline material with brick layer model. (a) Each component explicitly included in the circuit; (b) simplified equivalent circuit.

boundary model was assumed, wherein the model includes grain boundaries that are parallel or vertical to the applied field (Fig. 5). ${ }^{27)}$ In Fig. 5, L denotes the length of the sample, A the cross-sectional area of the sample, $d$ the edge length of a grain, and $\delta$ the thickness of the grain boundary. If $\delta<<\mathrm{d}$, the total length and the area of all samples for all the perpendicular (or series) grain boundaries is given by: $L_{\perp}=(\delta / d) L$ and $A_{\perp}=A$. Similarly, the total length and the area of all the parallel grain boundaries is given as $L_{\|}=L$ and $A_{\|}=(2 \delta / d) A$. Fig. 6 shows the RQ sub-circuit corresponding to an ideal polycrystalline material. The first (RQ) circuit consists of parallel grain boundaries and grain interiors, and the second RQ circuit consists of the only series grain boundaries. The third CPE represents the electrode reaction. The equivalent circuit of Fig. 6(a) can be simplified, as done in Fig. 6(b) by combining the two parallel resistances into one simple resistance. Similarly, two CPEs can be merged into one $Q$. If the value of $n$ is assumed to be the same, the entire pseudo-capacitance may be expressed as $Y_{g b \|}=Y_{b u l k}$ (Fig. 6(b)). In addition, as is known empirically, it was assumed that the low to mid frequency arc is related to the responses of grain boundaries in series with grain interior and the high frequency arc corresponds to the combined the response of grain interiors and parallel grain boundaries. Taking the specific conductivities of the grain boundary and the bulk are $\sigma_{g b}$ and $\sigma_{b u l k}$, respectively, and if the conduction in series and parallel grain boundaries follows the same mechanism, yield

$$
\frac{1}{R_{1}}=\frac{1}{R_{g b \|}}+\frac{1}{R_{b u l k}}=\frac{A}{L}\left\{\frac{2 \delta}{d} \sigma_{g b}+\sigma_{b u l k}\right\},
$$




$$
\frac{1}{R_{2}}=\frac{1}{R_{g b \|}}=\frac{A}{L}\left\{\frac{d}{\delta} \sigma_{g b}\right\}
$$
Defining $\sigma_{i}=L / A\left\{\frac{1}{R_{i}}\right\}$, Eqs. (2) and (3) may be rewritten
as follows:

$$
\begin{aligned}
& \sigma_{1}=\frac{2 \delta}{d} \sigma_{g b}+\sigma_{b u l k} \\
& \sigma_{2}=\frac{d}{\delta} \sigma_{g b}
\end{aligned}
$$

From an impedance measurement and an information of the macroscopic sample dimensions, $R_{1}$ and $R_{2}$ can be obtained at once from the Nyquist plot, but $\sigma_{g b}$ and $\sigma_{b u l k}$ cannot be obtained. However, the impact of the relative magnitudes $\sigma_{g b}$ and $\sigma_{b u l k}$ on the relative magnitudes of $R_{1}$ and $R_{2}$ can be handled easily under various limiting conditions. The ratio of $R_{1}$ to $R_{2}$ can be obtained from the ratio between $\sigma_{1}$ and $\sigma_{2}$.

$$
\frac{\sigma_{1}}{\sigma_{2}}=\frac{R_{2}}{R_{1}}=2\left(\frac{\delta}{d}\right)^{2}+\left(\frac{\delta}{d}\right) \frac{\sigma_{b u l k}}{\sigma_{g b}}
$$

As shown by the relation above, the ratio of $R_{1}$ and $R_{2}$ is related to both $\left(\sigma_{b u l k} / \sigma_{g b}\right)$ and $(\delta / \mathrm{d})$.

Case (1): $\sigma_{b u l k}>\sigma_{g b}$

For the situation where $\sigma_{b u l k}>\sigma_{g b}$, the material generally has a microstructure with $\delta<<$ d. Therefore, Eq. (6) is reduced to:

$$
\frac{\sigma_{1}}{\sigma_{2}}=\frac{R_{2}}{R_{1}}=\left(\frac{\delta}{d}\right) \frac{\sigma_{b u l k}}{\sigma_{g b}}
$$

Similarly, Eq. (4) is reduced to:

$$
\sigma_{1}=\sigma_{\text {bulk }} \text {. }
$$

Equation (8) is general assumption for impedance analysis to calculate bulk conductivity. The Nyquist plots of materials with $\sigma_{b u l k}>\sigma_{g b}$ will present two distinguishable arc. if 1) $R_{1}$ and $R_{2}$ are comparable in magnitude, and 2) the characteristic frequencies of the two domains are sufficiently different. Materials with typical microstructure ( $\delta / d$ $<1$ and $\sigma_{b u l k}>\sigma_{g b}>1$ ), will have $0.01<\mathrm{R}_{1}<\mathrm{R}_{2}<100$. Therefore, the first condition is met. The characteristic frequency of a material or a material domain is given by $w_{0}=1 / R C$, wherein $w_{0}$ is a unique characteristic of a material that is independent to the geometric factors. Alternatively, $w_{0}$ can be expressed as $1 / \rho \varepsilon \varepsilon_{0}$, wherein $\rho$ is a resistivity given by $1 /$ $\sigma, \varepsilon$ denotes the relative permittivity, and $\varepsilon_{0}$ denotes the vacuum permittivity. Therefore, the question whether $w_{0, b u l k}$ is significantly different from $w_{0, g b}$ or not is eventually turned to whether $\left(\rho_{g b} \varepsilon_{g b}\right)$ is significantly different from $\left(\rho_{\text {bulk }} \varepsilon_{\text {bulk }}\right)$ or not. While resistivities of materials cover many orders of magnitude, dielectric constant do not significantly changed. Hence, $\varepsilon_{b u l k} \sim \varepsilon_{g b}$ is a reasonable assumption. Therefore, the question is reduced to whether $\rho_{\text {bulk }}$ is significantly different from $\rho_{g b}$ or not. Since $\sigma_{b u l k}>\sigma_{g b}$ is first assumed, by definition $\rho_{g b}>\rho_{b u l k}$, which eventually implies $w_{0, \text { bulk }}>w_{0, g b}$. Consequently, two arc will be presented in the Nyquist plot, and the semicircle at the low frequency will correspond to the responses of grain boundaries. More specifically, the characteristic frequency of the high frequency arc is determined as a combined the dielectric response of the grain interiors and parallel grain boundaries. However, since the parallel grain boundaries have an unfavorable geometric structure (a small area, and a long length), their contribution of the parallel grain boundary to the capacitance is negligible. In a typical microstructure, if $\sigma_{b u l k}>\sigma_{g b}$, $\sigma_{b u l k}$ may be immediately obtained (Eq. 8). However, $\sigma_{g b}$ requires the information about the grain size and the grain boundary (Eq. 5). In absence of microstructural observations, only $d / \delta$ can be directly estimated. Each RQ sub-circuit has a capacitance value. It can be obtained from the resistance and the characteristic frequency, and the two variables, $\mathrm{Y}$ and $\mathrm{n}$ of $\mathrm{CPE}$ which characterize the constant phase element. Specifically,

$$
C=\frac{1}{R w_{0}}=Y^{(1 / n)} R^{\left(\frac{1}{n}-1\right)}
$$

As mentioned above, due to the unfavorable geometry of the parallel grain boundary, the dielectric constant $\mathrm{C}_{1}$ is related to the high frequency arc and it is entirely associated to the bulk. On the contrary, $\mathrm{C}_{2}$ is related to the low frequency arc and it is almost exclusively associated to the vertical (or series) grain boundary. Therefore, $\mathrm{C}_{1}$ and $\mathrm{C}_{2}$ may be expressed as in the following equations:

$$
\begin{aligned}
& C_{1} \approx C_{b u l k}=\frac{A}{L} \varepsilon_{b u l k} \varepsilon_{0}, \\
& C_{2} \approx C_{g b \perp}=\frac{A}{\bar{L}} \varepsilon_{g b} \varepsilon_{0}
\end{aligned}
$$

Since the overall dielectric characteristic of the bulk and the grain boundary is quite similar $\left(\varepsilon_{b u l k} \sim \varepsilon_{g b}\right)$,

$$
\frac{C_{2}}{C_{1}}=\frac{\delta}{d}
$$

Therefore, both the specific grain interior conductivity and the specific grain boundary conductivity can be measured by accurately using the relevant variables without microstructural observations.

\section{Case (2): $\sigma_{g b}>\sigma_{b u l k}$}

In this case, for those materials which have typical microstructures, $\delta / d$ no greater than $1 / 100$, no simplification to Eq. (6) can be applied. Eq. (2) is applied to determine the shape of the Nyquist plot in the following three different sub-cases:

$$
\text { (a) } \sigma_{g b} / \sigma_{b u l k}>\frac{d}{2 \delta}\left(R_{g b \|}<<R_{b u l k}\right)
$$

In this sub-case, $R_{g b \|}$ becomes dominant over $\mathrm{R}_{1}$; the ionic transport along the parallel grain boundary is preferred to 
the passage through the grain interiors

(b) $\sigma_{g b} / \sigma_{b u l k} \sim \frac{d}{2 \delta}\left(R_{g b \|} \sim R_{b u l k}\right)$

In this sub-case, since $R_{g b \|}$ is similar to $R_{b u l k}$, the ionic transport occurs through both the parallel grain boundary and the grain interiors.

$$
\text { (c) } \sigma_{g b} / \sigma_{b u l k}<<\frac{d}{2 \delta}\left(R_{g b \|}>>R_{b u l k}\right)
$$

The resistance $R_{1}$ will be dominated by $R_{\text {bulk }}$. Therefore, the assumptions for Eqs. (7) and (8) are valid. As assumed above, if the dielectric constants of bulk is similar to that of grain boundaries and $\mathrm{n}$ is close to 1 , the capacitance of the first RQ sub-circuit (Fig. 6(b)) may again be expressed as $\mathrm{C}_{1}$ $\sim \mathrm{C}_{\text {bulk }}$ in all the sub-cases. In the sub-cases (a) and (b), $\mathrm{R}_{1}$ is within a factor of two $R_{g b \|}$. However, in sub-case (c), $R_{1}$ is close to $R_{\text {bulk. }}$. In all the sub-cases, the second RQ circuit resistance, $R_{2}$, is much smaller than $R_{1}$. In sub-cases (a) and (b), $R_{2}$ may be expressed as in the following equation:

$$
R_{2}=R_{g b \perp}=2 R_{g b \|}\left(\frac{\delta}{d}\right)^{2} \approx 2 R_{1}\left(\frac{\delta}{d}\right)^{2}
$$

On the other hand, in sub-case (c), $R_{2}$ may be expressed as in Eq. (7). In all the sub-cases, the capacitance of the second RQ circuit may be expressed as in the following equation:

$$
C_{2}=C_{g b \perp}=\frac{1}{2} C_{g b \|}\left(\frac{d}{\delta}\right)^{2} \approx C_{1}\left(\frac{d}{\delta}\right)^{2}
$$

The final approximation was based on Eq. (12). Since the responses of the first and the second sub-circuits are different, in the case of $\sigma_{g b}>\sigma_{b u l k}$, the Nyquist plot theoretically exhibit two arcs. In contrast to what is generally observed results, the high frequency arc corresponds to the $R_{2} Q_{2}$ sub-circuit of the grain boundary in series with (or perpendicular to) grains, while low frequency arc corresponds to the combined $R_{1} Q_{1}$ sub-circuit of the parallel grain boundaries and grain interiors. This is the result of $\mathrm{R}_{2} \mathrm{C}_{2}<\mathrm{R}_{1} \mathrm{C}_{1}$ (Eqs. (4), (10), and (11)). However, a two arcs spectrum is not actually be obtained, because the radius of the high frequency arc, $R_{2}$, is several orders of smaller than low fre-

quency arc. For example, if $\frac{\sigma_{g b}}{\sigma_{b u l k}}<\frac{d}{2 \delta}$ and $\delta / d=1 / 100, \mathrm{R}_{2} /$ $\mathrm{R}_{1}=10^{-4}$. Consequently, the Nyquist plot will exhibit a single arc.

\section{Case (3): $\sigma_{g b} \sim \sigma_{b u l k}$}

For case (3), in which the grain boundary conductivity and the bulk conductivity of a material with typical microstructure are almost similar to those in case (1), $\sigma_{b u l k}>\sigma_{g b}$. Eq. (6)

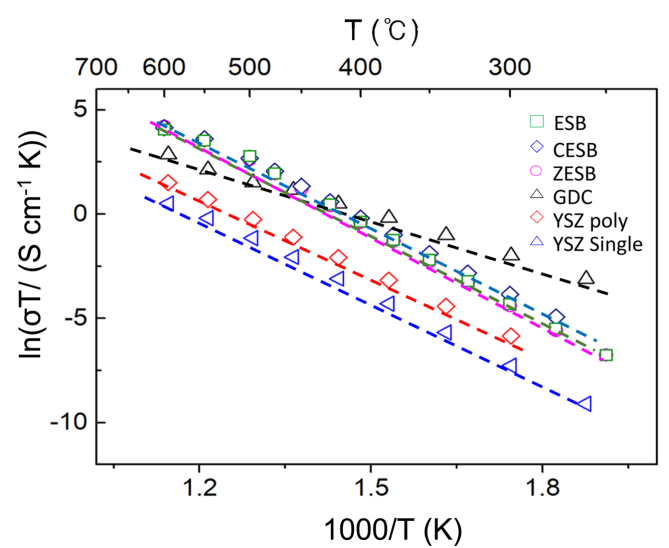

Fig. 7. Bulk ionic conductivity of all samples. Data are plotted in Arrhenius form.

is reduced to Eq. (7), and Eq. (4) may be expressed as Eq. (8). The case in which two arcs are obtained in the Nyquist plot is almost the same as case (1): if (i) $R_{1}$ is different from $\mathrm{R}_{2}$ by a factor of 10 to 1000 times, and (ii) $w_{0, \text { bulk }}$ is significantly different from $w_{0, g b}$. According to Eq. (7), The Raito $R_{2} / R_{1}$ is $1 / 100$ or smaller, for typical microstructures. The ratio is relatively small but not too small to be indistinguishable in the Nyquist plot. However, under the assumption of $\varepsilon_{b u l k} \sim \varepsilon_{g b}$, the characteristic frequencies, $w_{0, b u l k}$ and $w_{0, g b}$, are almost identical. As a result, only single arc will be observed. In addition, if $\sigma_{g b}$ is almost the same as $\sigma_{b u l k}$, the $\sigma_{1}$ obtained from the single arc will be within $2 \%$ of the $\sigma_{b u l k}$.

In summary, only for $\sigma_{b u l k}>\sigma_{g b}$, two arcs can be observed.

\subsection{Electrochemical Impedance Spectroscopy}

The ionic conductivity in the temperature range from 600 to $250^{\circ} \mathrm{C}$ was obtained by impedance analysis using the brick layer model. Since the electric conductivity of a ceramic material is thermally activated, the following Arrhenius equation was used:

$$
\sigma T=\sigma_{0} e^{-E_{\alpha} / k T}
$$

where $\mathrm{E}_{\mathrm{a}}$ denotes the activation energy needed for ionic conduction, $\sigma_{0}$ is the pre-exponential factor, and $\mathrm{k}$ is the Boltzmann constant. The activation energy was obtained from the Arrhenius plot (Fig. 7). As shown in Table 1, the grain boundary activation energy $(1.16 \mathrm{eV})$ of the YSZ polycrystals is higher than bulk activation energy $(1.06 \mathrm{eV})$. Similarly, for GDC polycrystals, the grain boundary activation energy $(0.97 \mathrm{eV})$ is higher than bulk activation energy

\begin{tabular}{|c|c|c|c|c|c|c|}
\hline & $\begin{array}{c}\text { polycrystalline } \\
\text { YSZ }\end{array}$ & $\begin{array}{c}\text { single crystal } \\
\text { YSZ }\end{array}$ & $\begin{array}{c}\text { polycrystalline } \\
\text { GDC }\end{array}$ & $\begin{array}{c}\text { polycrystalline } \\
\text { ESB }\end{array}$ & $\begin{array}{c}\text { polycrystalline } \\
\text { ZESB }\end{array}$ & $\begin{array}{c}\text { polycrystalline } \\
\text { CESB }\end{array}$ \\
\hline Bulk $E_{a}(e V)$ & 1.06 & 1.16 & 0.68 & 1.33 & 1.31 & 1.24 \\
\hline G.b $\mathrm{E}_{\mathrm{a}}(\mathrm{eV})$ & 1.16 & - & 0.97 & - & - & - \\
\hline
\end{tabular}
$(0.68 \mathrm{eV})$. In Fig. 8, an impedance spectra was obtained at low-temperature because the grain boundary arc can be

Table 1. Activation Energy of Each Sample 


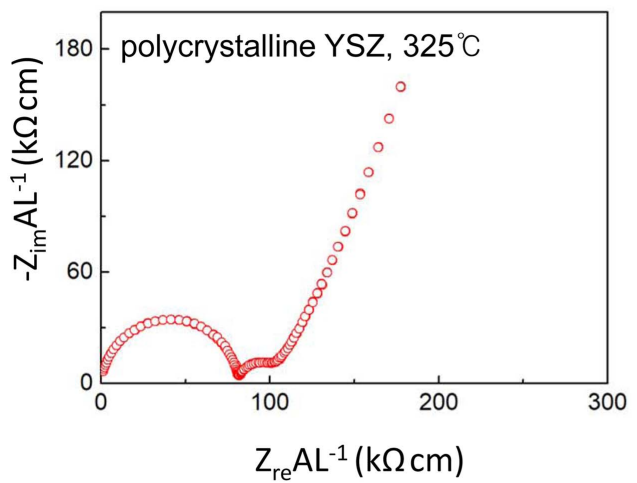

(a)

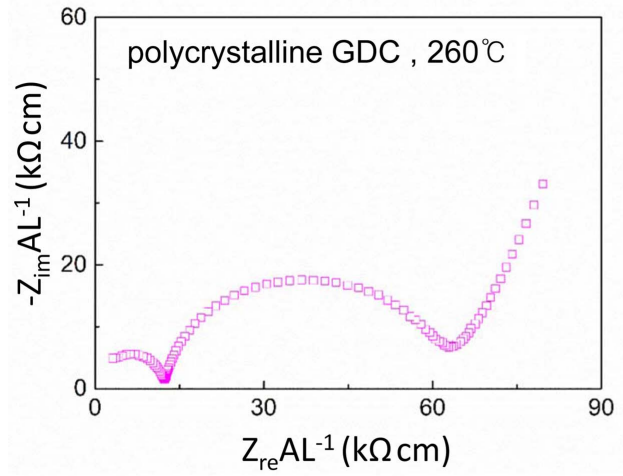

(c)

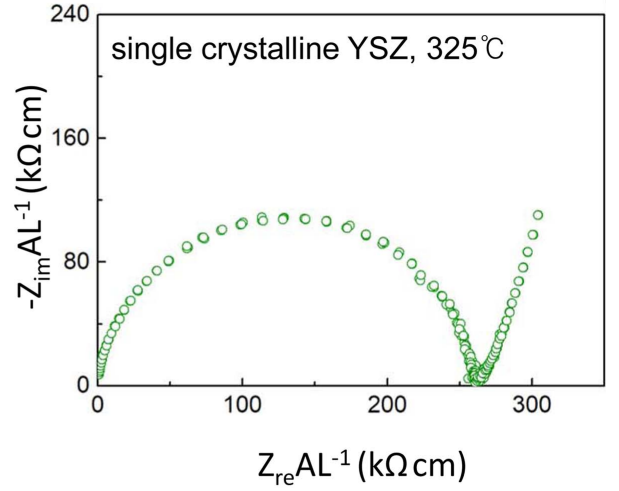

(b)

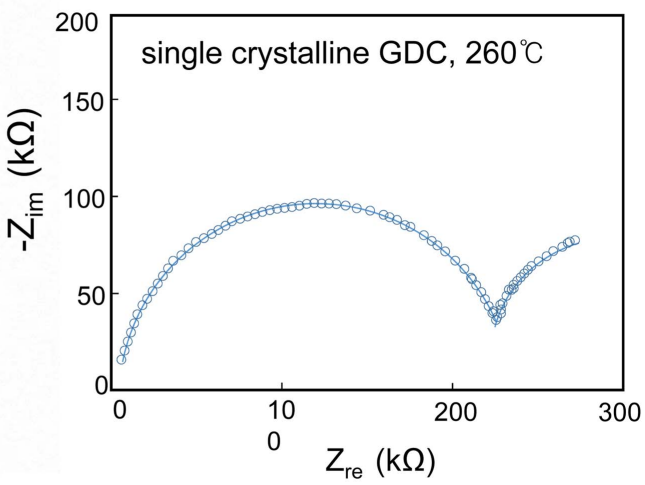

(d)

Fig. 8. (a-d) Nyquist impedance plots obtained from polycrystalline YSZ, single crystal YSZ, polycrystalline GDC, and single crystalline $\mathrm{Ce}_{0.69} \mathrm{Gd}_{0.31} \mathrm{O}_{2-\delta}$ (GDC) impedance spectra data; (d) is from ref. 30 .

obtained at a low temperature. Two arcs of the bulk and the grain boundaries in polycrystalline YSZ were obtained (Fig. 8(a)). Since two arcs, which represented in the polycrystalline, corresponds to Case $1\left(\sigma_{b u l k}>\sigma_{g b}\right)$ of the brick layer model. Only one arc corresponding to the bulk was obtained in the single crystal (Fig. 8(b)). Also, for polycrystalline GDC, two arc of the bulk and the grain boundary was obtained at $260^{\circ} \mathrm{C}$ in the Nyquist plot (Fig. 8(c)), which corresponds to Case $1\left(\sigma_{b u l k}>\sigma_{g b}\right)$. The impedance spectra data for single crystal GDC were obtained from the previous study because we cannot find GDC single crystal ${ }^{30)}$ As expected, only single arc is obtained the single crystal GDC because of the absence of the grain boundary. As can be seen in Fig. 9(d), (e), and (f), although ESB is polycrystalline material, grain boundary arc cannot be obtained in the Nyquist plot. ESB did not have a grain boundary blocking effect caused by the space charge layer because ESB has no aliovalent dopant which causes segregation at the grain boundaries, in contrast to acceptor doped zirconia and acceptor doped ceria (Fig. 1). We believed that CESB (Eq. (1b)) will have a grain boundary effect, because the segregation of $\mathrm{Ca}_{\mathrm{Bi}}^{\prime}$ at the grain boundary will make space charge layer similar to the case of acceptor doped zirconia and ceria. $^{12-15)}$ Likewise, we also believed that ZESB (Eq. (1a)) have a grain boundary effect because the segregation of $\mathrm{Ca}_{\mathrm{Bi}}^{\prime}$ at the grain boundary will cause a space charge layer.
However, only single arc, not two arcs is obtained in the Nyquist plot form both CESB and ZESB. This means that the blocking effect due to the space charge does not exist in $\delta-\mathrm{Bi}_{2} \mathrm{O}_{3}$. The resistance of the ESB at $275^{\circ} \mathrm{C}$ is $132.6 \mathrm{k} \Omega \mathrm{cm}$, but that of CESB is lower, $75.6 \mathrm{k} \Omega \mathrm{cm}$. The activation energy of CESB is $1.23 \mathrm{eV}$, which is also lower than that of ESB $(1.33 \mathrm{eV})$. The resistance of ZESB at $275^{\circ} \mathrm{C}$ is $147 \mathrm{k} \Omega \mathrm{cm}$, which is about $10 \%$ higher than that of ESB. The activation energy of ZESB is $1.31 \mathrm{eV}$, which was similar to that of ESB $(1.33 \mathrm{eV})$. The lower resistance and the lower activation of CESB may be due to the increased oxygen vacancy concentration resulting from acceptor doping. The increased resistance in ZESB probably is due to the decreased oxygen vacancy concentration resulting from donor doping. This result is consistent with a previous study conducted with Ystabilized $\delta-\mathrm{Bi}_{2} \mathrm{O}_{3}$ doped with $\mathrm{Zr}$ or $\mathrm{Ca} .{ }^{31)}$ The absence of the space charge effect in the cubic bismuth oxides, regardless of the acceptor or donor doping, may have been because of the high oxygen vacancy concentration of $\delta-\mathrm{Bi}_{2} \mathrm{O}_{3}$ itself $(25 \%) .{ }^{8)}$ The oxygen vacancy depletion in the space charge layer was proposed as the cause of the grain boundary blocking effect. ${ }^{2)}$ In $\delta-\mathrm{Bi}_{2} \mathrm{O}_{3}$, the oxygen vacancy depletion in the space charge layer may be negligible, because the oxygen vacancy concentration of $\delta-\mathrm{Bi}_{2} \mathrm{O}_{3}$ was much higher (25\%) than that of 8YSZ (oxygen vacancy concentration: $8 \%$ ) and GDC (oxygen vacancy concentration: $10 \%$ ). In addition, 


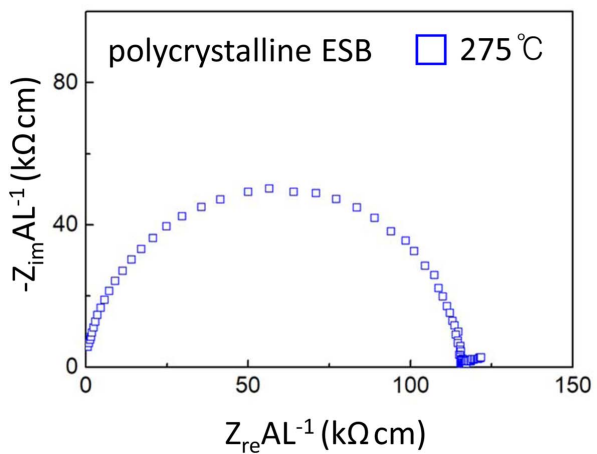

(a)

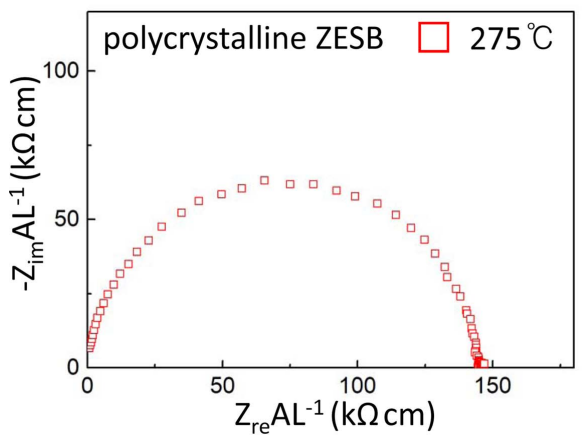

(b)

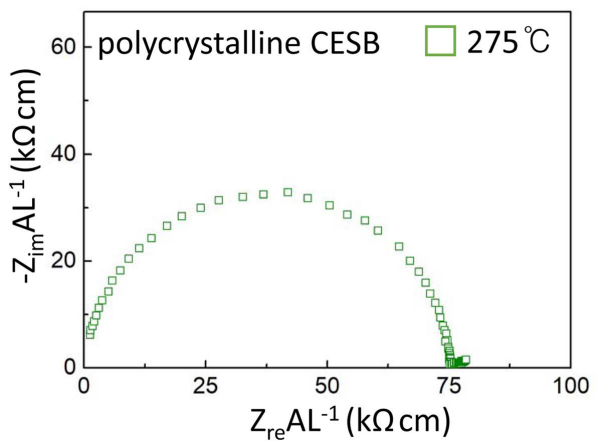

(c)

Fig. 9. (a-c) Impedance spectra from polycrystalline ESB, ZESB and CESB.

according to the brick layer model, the cases in which a single impedance arc is obtained are Case $2\left(\sigma_{b u l k} \sim \sigma_{g b}\right)$ and Case $3\left(\sigma_{b u l k}<\sigma_{g b}\right)$. Therefore, the grain boundary ionic conductivity of $\delta-\mathrm{Bi}_{2} \mathrm{O}_{3}$ should be similar to or higher than the bulk ionic conductivity. because of the high oxygen vacancy concentration and the high ionic conductivity at the grain boundary, $\delta-\mathrm{Bi}_{2} \mathrm{O}_{3}$ is almost free from the space charge effect, unlike other fluorite-type structure materials, thus it does not show the grain boundary blocking effect. An oxygen diffusion study of $\mathrm{Bi}_{2} \mathrm{O}_{3}$ conducted by oxygen tracer diffusion measurements demonstrated that Grain boundary ion diffusion in $\alpha-\mathrm{Bi}_{2} \mathrm{O}_{3}$ is three to four magntude higher than bulk at $600^{\circ} \mathrm{C}$, which is similar to the oxygen ion diffusion rate in bulk $\mathrm{Ce}_{0.7} \mathrm{Gd}_{0.3} \mathrm{O}_{1.85}{ }^{32)}$ In short, the main mechanism of the ionic conduction in $\delta-\mathrm{Bi}_{2} \mathrm{O}_{3}$ may be through the grain boundaries. To prove this theory more directly, fur- ther studies need to be conducted to observe the dopant segregation at the grain boundaries of ZESB and CESB through TEM and EELS and the space charge potential through KFM, which will demonstrate more direct experimental evidences that Space charge effect is negligible in cubic bismuth oxide.

\section{Conclusions}

In this work, an electrochemical impedance analysis was performed to investigate the grain boundary blocking effect due to the space charge in fluorite-type structured YSZ, GDC, ESB, ZESB, and CESB. The results showed that two distinguishable arcs were obtained in the Nyquist plot of polycrystalline YSZ and GDC, which confirmed the presence of the grain boundary blocking effect. The single-crystal materials with no grain boundary have only one arc, as expected. However, polycrystalline ESB and ZESB, and CESB have only one arc, indicating the absence of the grain boundary blocking effect caused by the space charge. The absence of the blocking effect in $\delta-\mathrm{Bi}_{2} \mathrm{O}_{3}$ may be because the intrinsic oxygen vacancy concentration in $\delta-\mathrm{Bi}_{2} \mathrm{O}_{3}$ is as high as $25 \%$ due to crystal structure. In addition, by applying the brick layer model for the impedance analysis, we denmonstrate that grain boundary ionic conductivity of $\delta-\mathrm{Bi}_{2} \mathrm{O}_{3}$ is or even higher than bulk ionic conductivity $\left(\sigma_{b u l k} \leq \sigma_{b g}\right)$. Therefore, in this study we indirectly demonstrated that for $\delta$ - $\mathrm{Bi}_{2} \mathrm{O}_{3}$, grain boundary may be a main ionic conductivity path unlike other fluorite-type materials and grain boudnary blocking effect is absence using impedance analysis.

\section{Acknowledgements}

This work was supported by the National Research Foundation of Korea (Grant NO. 2014R1A4A1003712 (Basic Research Laboratory Project)) and the Ministry of Trade, Industry and Energy (Grant NO. 10065691). The authors acknowledge Hye-Sung Kim and Hyewon Ryoo for their fruitful comments.

\section{REFERENCES}

1. E. D. Wachsman and K. T. Lee, "Lowering the Temperature of Solid Oxide Fuel Cells," Science, 334 [6058] 935-39 (2011).

2. A. M. Azad, S. Larose, and S. A. Akbar, "Bismuth Oxidebased Solid Electrolytes for Fuel Cells," J. Mater. Sci., 29 [16] 4135-51 (1994).

3. V. V. Kharton, F. M. B. Marques, and A. Atkinson, "Transport Properties of Solid Oxide Electrolyte Ceramics: A Brief Review," Solid State Ionics, 174 [1] 135-49 (2004).

4. P. Shuk, H. D. Wiemhöfer, U. Guth, W. Göpel, and M. Greenblatt, "Oxide Ion Conducting Solid Electrolytes based on $\mathrm{Bi}_{2} \mathrm{O}_{3}$," Solid State Ionics, 89 [3-4] 179-96 (1996).

5. N. M. Sammes, G. A. Tompsett, H. Nafe, and F. Aldinger, "Bismuth based Oxide Electrolytes-Structure and Ionic Conductivity," J. Eur. Ceram. Soc., 19 [10] 1801-26 (1999). 
6. M. Mogensen, N. M. Sammers, and G. A. Tompsett, "Physical, Chemical, and Electrochemical Properties of Pure and Doped Ceria," Solid State Ionics, 129 [1] 63-94 (2000).

7. B. C. H. Steele, "Material Science and Engineering: The Enabling Technology for the Commercialisation of Fuel Cell Systems," J. Mater. Sci., 36 [5] 1053-68 (2001).

8. P. D. Battle, C. R. A. Catlow, J. W. Heap, and L. M. Moroney, "Structural and Dynamical Studies of $\delta-\mathrm{Bi}_{2} \mathrm{O}_{3}$ Oxide-Ion Conductors II. A Structural Comparison of $\left(\mathrm{Bi}_{2} \mathrm{O}_{3}\right)_{1-\mathrm{x}}\left(\mathrm{M}_{2} \mathrm{O}_{3}\right)_{\mathrm{x}}$ for $\mathrm{M}=\mathrm{Y}$, Er and Yb," J. Solid State Chem., 67 [1] 42-50 (1987).

9. X. Guo, W. Sigle, and J. Maier, "Blocking Grain Boundaries in Yttria-Doped and Undoped Ceria Ceramics of High Purity," J. Am. Ceram. Soc., 86 [1] 77-87 (2003).

10. H. J. Avila-Paredes, K. Choi, C. T. Chen, and S. Kim, "Dopant-Concentration Dependence of Grain-Boundary Conductivity in Ceria: A Space-Charge Analysis," J. Mater. Chem., 19 [27] 4837-42 (2009).

11. X. Guo and J. Maier, "Grain Boundary Blocking Effect in Zirconia: A Schottky Barrier Analysis," J. Electrochem. Soc., 148 [3] E121-26 (2001).

12. X. Guo, W. Sigle, J. Fleig, and J. Maier, "Role of Space Charge in the Grain Boundary Blocking Effect in Doped Zirconia," Solid State Ionics, 154 555-61 (2002).

13. N. M. Beekmans and L. Heyne, "Correlation between Impedance, Microstructure and Composition of CalciaStabilized Zirconia," Electrochim. Acta, 21 [4] 303-10 (1976).

14. S. P. S. Badwal, "Grain Boundary Resistivity in Zirconiabased Materials: Effect of Sintering Temperatures and Impurities," Solid State Ionics, 76 [1-2] 67-80 (1995).

15. M. Aoki, Y. M. Chiang, I. Kosacki, L. Lee, H. Tuller, and Y. Liu, "Solute Segregation and Grain-Boundary Impedance in High-Purity Stabilized Zirconia," J. Am. Ceram. Soc., 79 [5] 1169-80 (1996).

16. X. Guo and R. Waser, "Electrical Properties of the Grain Boundaries of Oxygen Ion Conductors: Acceptor-doped Zirconia and Ceria," Prog. Mater. Sci., 51 [2] 151-210 (2006).

17. S. Kim, J. Fleig, and J. Maier, "Space Charge Conduction: Simple Analytical Solutions for Ionic and Mixed Conductors and Application to Nanocrystalline Ceria," Phys. Chem. Chem. Phys., 5 [11] 2268-73 (2003).

18. Y. Lei, Y. Ito, N. D. Browning, and T. J. Mazanec "Segregation Effects at Grain Boundaries in Fluorite-Structured Ceramics," J. Am. Ceram. Soc., 85 [9] 2359-63 (2002).

19. W. Lee, H. J. Jung, M. H. Lee, Y. B. Kim, J. S. Park, R. Sinclair, and F. B. Prinz, "Oxygen Surface Exchange at Grain Boundaries of Oxide Ion Conductors," Adv. Funct.
Mater., 22 [5] 965-71 (2012).

20. P. Duran, J. R. Jurado, C. Moure, N. Valverde, and B. C. $\mathrm{H}$. Steele, "High Oxygen Ion Conduction in Some $\mathrm{Bi}_{2} \mathrm{O}_{3}$ $\mathrm{Y}_{2} \mathrm{O}_{3}\left(\mathrm{Er}_{2} \mathrm{O}_{3}\right)$ Solid Solutions," Mater. Chem. Phys., 18 [3] 287-94 (1987).

21. M. F. Yan, R. M. Cannon, and H. K. Bowen, "Space Charge, Elastic Field, and Dipole Contributions to Equilibrium Solute Segregation at Interfaces," J. Appl. Phys., 54 [2] 764-78 (1983).

22. R. D. Shannon, "Revised Effective Ionic Radii and Systematic Studies of Interatomic Distances in Halides and Chalcogenides," Acta Cryst. A, 32 751-67 (1976).

23. F. A. Kröger and H. J. Vink, "Relations betwwen the Concentration of Imperfections in Crystalline Solids," pp. 307435 in Solid State Physics, Vol. 3, Ed. by F. Seitz, \& D. Turnbull, Academic press, New York, 1956.

24. H. A. Harwig and A. G. Gerards, "The Polymorphism of Bismuth Sesquioxide," Thermochim. Acta, 28 [1] 121-31 (1979).

25. M. J. Verkerk, K. Keizer, and A. J. Burggraaf, "High Oxygen Ion Conduction in Sintered Oxides of the $\mathrm{Bi}_{2} \mathrm{O}_{3}-\mathrm{Er}_{2} \mathrm{O}_{3}$ System," J. Appl. Electrochem., 10 [1] $81-90$ (1980).

26. N. Bonanos, B. C. H. Steele, and E. P. Butler, Impedance Spectroscopy; $1^{\text {st }}$ ed., pp. 191-238, Ed. by J. R. MacDonald, Wiley and Sons, New York, 1988.

27. H. Näfe, "Ionic Conductivity of $\mathrm{ThO}_{2}$-and $\mathrm{ZrO}_{2}$-based Electrolytes between 300 and 2000 K," Solid State Ionics, 13 [3] 255-63 (1984).

28. G. M. Christie and F. P. F. Van Berkel, "MicrostructureIonic Conductivity Relationships in Ceria-Gadolinia Electrolytes," Solid State Ionics, 83 [1-2] 17-27 (1996).

29. S. M. Haile, L. W. David, and J. Campbell, "The Role of Microstructure and Processing on the Proton Conducting Properties of Gadolinium-doped Barium Cerate," J. Mater. Res., 13 [6] 1576-95 (1998).

30. E. Ruiz-Trejo, J. D. Sirman, Y. M. Baikov, and J. A Kilner, "Oxygen Ion Diffusivity, Surface Exchange and Ionic Conductivity in Single Crystal Gadolinia Doped Ceria," Solid State Ionics, 113 565-69 (1998).

31. K. Z. Fung and A. V. Virkar, "Phase Stability, Phase Transformation Kinetics, and Conductivity of $\mathrm{Y}_{2} \mathrm{O}_{3}-\mathrm{Bi}_{2} \mathrm{O}_{3}$ Solid Electrolytes Containing Aliovalent Dopants," J. Am. Ceram. Soc., 74 [8] 1970-80 (1991).

32. R. D. Bayliss, S. N. Cook, S. Kotsantonis, R. J. Chater, and J. A. Kilner "Oxygen Ion Diffusion and Surface Exchange Properties of the $\alpha$-and $\delta$-phases of $\mathrm{Bi}_{2} \mathrm{O}_{3}$, , Adv. Energy Mater., 4 [10] 1301575 (2014). 